\title{
LIGNANAS E TRITERPENOS DO EXTRATO CITOTÓXICO DE ERIOPE BLANCHETII
}

\author{
Juceni P. David *, Eliana F. da Silva, Daniel L. de Moura \\ Faculdade de Farmácia, Universidade Federal da Bahia, 40170-290 Salvador - BA \\ Maria Lenise da S. Guedes \\ Instituto de Biologia, Universidade Federal da Bahia, 40170-290 Salvador - BA \\ Roberval de J. Assunção e Jorge M. David \\ Instituto de Química, Universidade Federal da Bahia, 40170-290 Salvador - BA
}

Recebido em 6/7/00; aceito em 21/3/01

\begin{abstract}
LIGNANS AND TRITERPENES FROM CYTOTOXIC EXTRACT OF ERIOPE BLANCHETII. This phytochemical study performed with the cytotoxic chloroformic extract of Eriope blanchetii (Benth.) Harley was the first work with this species and describes from aerial parts the isolation of two lignans of podophylotoxin type named $\beta$-peltatin and $\alpha$-peltatin. Besides them it was obtained four triterpenes; oleanolic acid, ursolic acid, $2 \alpha, 3 \beta$-dihydroxy-urs-12-en-28-olic acid, $2 \alpha, 3 \beta, 19 \alpha$-trihydroxy-urs-12-en-28-olic acid, named tormentic acid and $3 \beta$-glucosyl-sitosterol. The compounds were identified by analysis of their spectral data.
\end{abstract}

Keywords: Lamiaceae; Eriope blanchetii; lignans; triterpenes.

\section{INTRODUÇÃO}

Dentre as lignanas, a podofilotoxina possui um efeito antitumoral comprovado cientificamente. $\mathrm{O}$ maior efeito citotóxico da podofilotoxina é a inibição celular na metafase, detendo a duplicação dos cromossomos, resultando na retenção da divisão celular no estado mitótico do ciclo celular. Porém, devido ao fato destes efeitos atingirem tanto células normais como células cancerígenas, o seu uso tem sido limitado. No entanto, os derivados da podofilotoxina, como o etoposido e teniposido, não exibem nenhum efeito intracelular, mas induzem a quebra das ligações simples e duplas do DNA ${ }^{1}$. O etoposido (4-dimetil -7-[4,6- $O$-etilideno $\beta$-D-glicopiranosil epipodofilotoxina]) e o teniposido (4-dimetil-7-[4,6-O-tenilideno- $\beta$-D-glicopiranosil epipodofilotoxina]) são dois derivados semi-sintéticos que foram desenvolvidos em 1970 na tentativa de preparar derivados da podofilotoxina que não possuíssem efeito tóxico gastro-intestinal igual ao desta substância ${ }^{2}$. Nos quinze anos seguintes o etoposido e o teniposido foram introduzidos no tratamento clínico e estas drogas têm tido uma participação muito importante no tratamento quimioterápico do câncer. $\mathrm{O}$ desenvolvimento do etoposido e do teniposido como agentes antitumorais, representa um modelo para investigação sistemática de produtos naturais, e tem liderado a descoberta de uma nova classe de drogas antitumorais ${ }^{2}$. Além do mais, o desenvolvimento de novos agentes anticancerígenos, tais como etoposido e paclitaxel, proporcionou não somente a descoberta de drogas anticancerígenas derivadas de plantas, como também contribuiu para o aumento do conhecimento da biologia do câncer ${ }^{1,2}$.

$\mathrm{O}$ gênero Eriope pertencente a família Lamiaceae (Labiatae) é nativo das regiões tropical e subtropical da América do Sul e possui cerca de 20 espécies, sendo que 18 destas estão restritas ao território brasileiro. No Brasil, estas espécies distribuem-se principalmente em áreas de campo rupestre em Minas Gerais, Bahia, Goiás e estados vizinhos ${ }^{3,4}$.

Este é o primeiro relato na literatura do estudo fitoquímico e da citotoxicidade apresentada pelo extrato clorofórmico das partes aéreas de Eriope blanchetii (Benth.) Harley, bem como do isolamento das substâncias ativas presentes neste extrato. Neste

\footnotetext{
*e-mail: juceni@ufba.br
}

trabalho é descrito o isolamento das substâncias responsáveis pela atividade citotóxica $\left(\mathrm{IC}_{50} 16 \mu \mathrm{g} / \mathrm{mL}\right)$ observada para o extrato $\mathrm{CHCl}_{3}$ das partes aéreas de E. blanchetii. Assim, foram isolados quatro ácidos triterpênicos na forma de seus derivados metilados, os ácidos ursólico (1a), oleanólico (2a), ácido $2 \alpha, 3 \beta$ diidroxi-urs-12-en-28-ólico (5a) e ácido $2 \alpha, 3 \beta, 19 \alpha$-triidroxi-urs12-en-28-ólico (6a), conhecido como ácido tormentico, bem como o fitosterol, $3 \beta$-glicosil-sitosterol. Além destas substâncias, neste estudo também foram isoladas as lignanas $\beta$-peltatina (3) e $\alpha$-peltatina (4). A identificação estrutural das substâncias foi realizada por meio da análise de dados espectrométricos usuais.<smiles>[R]OC(=O)C12CCC(C)(C)CC1C1=CCC3[C@]4(C)CC[C@H](O)C(C)(C)[C@@H]4CC[C@]3(C)[C@]1(C)CC2</smiles>

1: $\mathrm{R}=\mathrm{H}$ 1a: $\mathrm{R}=\mathrm{CH}_{3}$

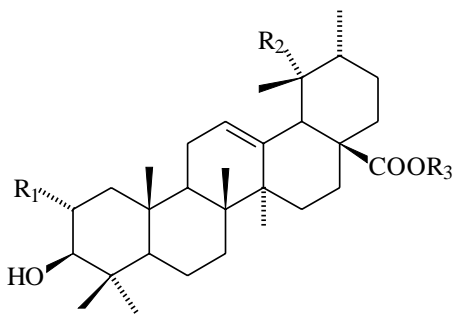

2: $\mathrm{R}_{1}=\mathrm{R}_{2}=\mathrm{R}_{3}=\mathrm{H}$

2a: $\mathrm{R}_{1}=\mathrm{R}_{2}=\mathrm{H}, \mathrm{R}_{3}=\mathrm{HCH}_{3}$ 5: $\mathrm{R}_{1}=\mathrm{OH}, \mathrm{R}_{2}=\mathrm{R}_{3}=\mathrm{H}$ 5a: $\mathrm{R}_{1}=\mathrm{OH}, \mathrm{R}_{2}=\mathrm{H} ; \mathrm{R}_{3}=\mathrm{CH}_{3}$ 6: $\mathrm{R}_{1}=\mathrm{OH}, \mathrm{R}_{2}=\mathrm{OH} ; \mathrm{R}_{3}=\mathrm{H}$ 6a: $\mathrm{R}_{1}=\mathrm{OH}, \mathrm{R}_{2}=\mathrm{OH} ; \mathrm{R}_{3}=\mathrm{CH}_{3}$ 
Tabela 1. Dados de $\mathrm{RMN}$ de ${ }^{13} \mathrm{C}$ dos derivados metilados dos triterpenos $2 \mathbf{a}, 5 \mathbf{a}$ e $\mathbf{6 a}$

\begin{tabular}{|c|c|c|c|}
\hline \multirow[t]{2}{*}{ Carbonos } & \multicolumn{3}{|c|}{ Substâncias } \\
\hline & $2 a$ & $5 \mathbf{a}$ & $6 a$ \\
\hline 1 & 38,8 & 46,6 & 46,3 \\
\hline 2 & 27,3 & 69,0 & 68,9 \\
\hline 3 & 78,8 & 83,9 & 83,9 \\
\hline 4 & 38,8 & 39,1 & 39,2 \\
\hline 5 & 55,4 & 55,3 & 55,2 \\
\hline 6 & 18,4 & 18,3 & 18,4 \\
\hline 7 & 33,0 & 32,8 & 32,6 \\
\hline 8 & 39,6 & 39,5 & 39,9 \\
\hline 9 & 47,5 & 47,5 & 47,1 \\
\hline 10 & 37,0 & 38,2 & 38,2 \\
\hline 11 & 23,3 & 23,4 & 23,7 \\
\hline 12 & 125,5 & 125,3 & 129,4 \\
\hline 13 & 138,0 & 138,2 & 138,1 \\
\hline 14 & 42,0 & 42,1 & 41,2 \\
\hline 15 & 28,2 & 28,0 & 28,1 \\
\hline 16 & 24,3 & 24,2 & 25,4 \\
\hline 17 & 48,1 & 48,1 & 47,9 \\
\hline 18 & 52,8 & 52,8 & 53,2 \\
\hline 19 & 39,1 & 39,0 & 73,2 \\
\hline 20 & 38,8 & 38,8 & 41,9 \\
\hline 21 & 30,7 & 30,6 & 26,0 \\
\hline 22 & 36,7 & 36,6 & 37,4 \\
\hline 23 & 28,2 & 28,6 & 28,6 \\
\hline 24 & 15,2 & 16,7 & 16,1 \\
\hline 25 & 15,7 & 16,8 & 16,5 \\
\hline 26 & 16,9 & 16,9 & 16,6 \\
\hline 27 & 23,6 & 23,6 & 24,5 \\
\hline 28 & 177,7 & 178,1 & 178,4 \\
\hline 29 & 16,9 & 17,0 & 27,4 \\
\hline 30 & 21,2 & 21,2 & 16,7 \\
\hline $\mathrm{OCH}_{3}$ & 51,6 & 51,5 & 51,6 \\
\hline
\end{tabular}

\section{PARTE EXPERIMENTAL}

Os espectros de RMN foram obtidos em espectrômetro Gemini 300 da Varian, sendo utilizado o sinal do solvente como referencial interno. Os espectros de massas foram obtidos por meio de injeção direta no detetor de massas da HP modelo 5973.

As partes aéreas de um espécimen Eriope blanchetii $(2,4 \mathrm{~kg})$ foram coletadas na restinga do Parque Metropolitano da Lagoa do Abaeté (Salvador, BA). O referencial da espécie encontra-se depositado no Herbário Alexandre Leal Costa do Instituto de Biologia da UFBA sob n ${ }^{\circ} 045599$.

$\mathrm{Na}$ realização do teste de letalidade de Artemia salina com as substâncias isoladas foi utilizada a metodologia modificada por Serrano e cols ${ }^{5}$. Parte da fase clorofórmica $(5 \mathrm{mg})$ foi submetida a avaliação da citotoxicidade in vitro em oito linhagens de células de acordo com o protocolo da University of Illinois at Chicago ${ }^{6}$ (Tabela 2).

O material vegetal após ter sido seco e moído foi macerado com $\mathrm{MeOH}$ e, o extrato metanólico bruto obtido após concentração, foi particionado sucessivamente entre hexano: $\mathrm{MeOH} / \mathrm{H}_{2} \mathrm{O}$ (9:1), $\mathrm{CHCl}_{3}: \mathrm{MeOH} / \mathrm{H}_{2} \mathrm{O}$ (6:4) e AcOEt: $\mathrm{H}_{2} \mathrm{O}$ (6:4). Assim, a fase clorofórmica $(7,65 \mathrm{~g})$ foi submetida a fracionamento em coluna cromatográfica sobre sílica gel usando misturas de $\mathrm{CHCl}_{3} /$ AcOEt em ordem crescente de polaridade e as frações obtidas purificadas através de diferentes tipos de cromatografia, além de permeação em Sephadex LH-20. Para promover a purificação dos ácidos triterpênicos, foi realizada a metilação das frações contendo os ácidos triterpênicos, com diazometano gerado in situ. Deste modo, após metilação, as frações eluídas com $\mathrm{CHCl}_{3} /$ AcOEt (8:2) foram reunidas e purificadas por meio de CC usando hexano/AcOEt (9:1) fornecendo o éster metílico do ácido oleanólico $(\mathbf{1 a} ; 2,5 \mathrm{~g})$ e do ácido ursólico $(\mathbf{2 a} ; 28,0 \mathrm{mg})$. A fração eluída com $\mathrm{CHCl}_{3} / \mathrm{AcOEt}$ (7:3) foi submetida a nova CC utilizando como eluentes, misturas de éter de petróleo/acetona e metanol em ordem crescente de polaridade. A fração eluída com éter de petróleo/acetona (1:1) foi purificada por meio de permeação em CC sob Sephadex LH-20 utilizando como eluente $\mathrm{MeOH} / \mathrm{CHCl}_{3}$ (4:1) fornecendo $\beta$-peltatina (3) (18,3 mg). Enquanto que, a fração eluída com $\mathrm{MeOH}$, foi submetida a CCDP usando como eluente $\mathrm{CHCl}_{3} / \mathrm{AcOEt}$ (1:1) forneceu $\alpha$-peltatina (4) $(20,1 \mathrm{mg})$. A última fração eluída com $\mathrm{MeOH}$ da CC principal do extrato $\mathrm{CHCl}_{3}$ foi metilada com $\mathrm{CH}_{2} \mathrm{~N}_{2}$ e fracionada em coluna sob pressão de silica gel $60 \mathrm{H}$ usando como eluente hexano/AcOEt (1:1), fornecendo os ésteres metílicos 5a $(10,5$ $\mathrm{mg})$ e $\mathbf{6 a}(25,3 \mathrm{mg})$, além de $\beta$-glicosil-sitosterol $(35,1 \mathrm{mg})$.<smiles>[R20]Oc1c(OC)cc([C@@H]2c3cc4c(c(O[R])c3C[C@@H]3COC(=O)[C@@H]32)OCO4)cc1OC</smiles>

$\beta$-Peltatina (3). $\mathrm{C}_{22} \mathrm{H}_{22} \mathrm{O}_{8}$. EM $\mathrm{m} / z$ (int. rel): 414 (100), 399 (5), 383 (7), 369 (10), 355 (11), 234 (5), 339 (5), 340 (5), 324 (5), 303 (10), 299 (11), 298 (8), 285 (7), 269 (5), 264 (5), 257 (8), 255 (9), 248 (13), 246 (14), 189 (36), 119 (26). RMN de ${ }^{1} \mathrm{H}\left[300 \mathrm{MHz}, \mathrm{CDCl}_{3}, \delta(\mathrm{ppm})\right.$, multiplicidade, J (Hz)]: $\delta 6,20$ $(s, \mathrm{H}-2) ; 3,17$ e $2,66(d d, \mathrm{~J}=13,7$ e $3,2 \mathrm{~Hz}, \mathrm{H}-7) ; 2,66(s l$, $\mathrm{H}-8) ; 3,81(m, \mathrm{H}-9)$ e $4,46(d d, \mathrm{~J}=6,9 \mathrm{~Hz}$ e indt., $\mathrm{H}-9) ; 6,33(s$, H-2'); 6,33 ( $\left.s, \mathrm{H}-6^{\prime}\right) ; 4,57$ ( $\left.d, \mathrm{~J}=3,1 \mathrm{~Hz}, \mathrm{H}-7^{\prime}\right) ; 2,66$ (H-8'); 5,95 $\left(s l, \mathrm{O}_{2} \mathrm{CH}_{2}\right) ; 3,76\left(2 \mathrm{CH}_{3} \mathrm{O}\right) ; 3,81\left(\mathrm{CH}_{3} \mathrm{O}\right) . \mathrm{RMN}$ de ${ }^{13} \mathrm{C}[75$ $\left.\mathrm{MHz}, \mathrm{CDCl}_{3}, \delta(\mathrm{ppm})\right]: \delta 137,1^{*}(\mathrm{C}-1) ; 103,5(\mathrm{C}-2) ; 152,5$ (C-3); 130,9 (C-4); 136,3* (C-5); 118,2 (C-6); 26,7 (C-7); 43,9 (C-8); 72,4 (C-9); 131, ${ }^{*}\left(\mathrm{C}-1^{\prime}\right) ; 108,4\left(\mathrm{C}-2^{\prime}\right) ; 152,5$ (C-3');

Tabela 2. Atividade citotóxica do extrato clorofórmico de Eriope blanchetii ${ }^{a}$

\begin{tabular}{ccccccccc}
\hline \multicolumn{7}{c}{ Linhagem Celular $^{\mathrm{b}}$} \\
\hline & $\mathrm{ASK}$ & $\mathrm{BC} 1$ & $\mathrm{Lu} 1$ & $\mathrm{Col} 2$ & $\mathrm{~KB}$ & $\mathrm{~KB}-\mathrm{V} 1(+\mathrm{VLB})$ & $\mathrm{KB}-\mathrm{V} 1(-\mathrm{VLP})$ & LNCaP \\
Ext. $\mathrm{CHCl}_{3}$ & + & $>20$ & $>20$ & $>20$ & 16.1 & $>20$ & $>20$ & $>20$ \\
\hline
\end{tabular}

$\overline{{ }^{a}\left(\mathrm{IC}_{50} \mathrm{em} \mu \mathrm{g} / \mathrm{mL}\right)} ;{ }^{b} \mathrm{ASK}=$ astrocitoma humano; BC1 = câncer humano de mama; Lu1 = câncer humano de pulmão; Col2 = câncer humano de cólon; $\mathrm{KB}$ = câncer humano/carcicoma; KB-V1 (+VLB) = resistente à vinblastina (VLB)-KB com $1 \mu \mathrm{g} / \mathrm{mL}$ VLB; KB$\mathrm{V} 1(-\mathrm{VLB})=$ Resistente à VLB - KB sem VLB; LNCaP = câncer de próstata dependente de hormônio.

\footnotetext{
* Valores podem estar trocados
} 
132,9* (C-4'); 152,5 (C-5'); 108,4 (C-6'); 32,2 (C-7'); 47,4 (C-8'); 175,1 (C-9'); 56,0 (2- $\left.\mathrm{OCH}_{3}\right), 60,7\left(\mathrm{OCH}_{3}\right) ; 101,7\left(\mathrm{O}_{2} \mathrm{CH}_{2}\right)$.

$\alpha$-Peltatina (4). $\mathrm{C}_{21} \mathrm{H}_{20} \mathrm{O}_{8}$. EM m/z (int. rel): 400 (100), 385 (3), 370 (4), 355 (20), 342 (10), 341 (27), 340 (15), 315 (12), 285 (18), 246 (31), 201 (14), 115 (11). RMN de ${ }^{1} \mathrm{H}[300 \mathrm{MHz}$, $\mathrm{CD}_{3} \mathrm{OD}, \delta(\mathrm{ppm})$, multiplicidade, J (Hz)]: $\delta$ 6,08 ( $\left.s, \mathrm{H}-2\right) ; 3,13$ $(d d, \mathrm{~J}=4,3$ e $14,0 \mathrm{~Hz}, \mathrm{H}-7)$ e $2,40(d d, \mathrm{~J}=4,3$ e $10,0 \mathrm{~Hz}, \mathrm{H}-7)$; $3,92(d d, \mathrm{~J}=8,4$ e $1,6 \mathrm{~Hz}, \mathrm{H}-9)$ e $4,44(d d, \mathrm{~J}=9,6 \mathrm{~Hz}$ e indt., H-9); 6,34 ( $\left.\left.s, \mathrm{H}-2^{\prime}\right) ; 6,34\left(s, \mathrm{H}-6{ }^{\prime}\right) ; 4,44(s l, \mathrm{H}-7)^{\prime}\right) ; 2,60(s l$, $\mathrm{H}-8$ '); 5,86 (sl, $\left.\mathrm{O}_{2} \mathrm{CH}_{2}\right) ; 3,69\left(2 \mathrm{CH}_{3} \mathrm{O}\right)$.

RMN de ${ }^{13} \mathrm{C}$ [75 MHz, $\left.\mathrm{CD}_{3} \mathrm{OD}, \delta(\mathrm{ppm})\right]: \delta 136,3^{*}(\mathrm{C}-1)$; 103,5 (C-2); 152,6 (C-3); 132,9 (C-4); 136,9* (C-5); 118,2 (C-6); 32,0 (C-7); 44,0 (C-8); 72,4 (C-9); 132,9 (C-1'); 108,3 (C-2'); 148,0 (C-3'); 131,9 (C-4'); 148,0 (C-5'); 108,3 (C-6'); 32,3 (C-7'); 47,4 (C-8'); 175,1 (C-9'); $56,7\left(2-\mathrm{OCH}_{3}\right) ; 102,3$ $\left(\mathrm{O}_{2} \mathrm{CH}_{2}\right)$

Diacetil- $\alpha$-peltatina (4a). RMN de ${ }^{1} \mathrm{H}\left[300 \mathrm{MHz}, \mathrm{CDCl}_{3}, \delta\right.$ (ppm), multiplicidade, $\mathrm{J}(\mathrm{Hz})]: \delta \quad 6,43(s, \mathrm{H}-2) ; 2,38(d d, \mathrm{~J}=2,4$ e $10,9 \mathrm{~Hz}$,$) e 2,93(d d, \mathrm{~J}=5,0$ e $15,9 \mathrm{~Hz}, \mathrm{H}-7) ; 3,85(d d$, $\mathrm{J}=10,0$ e $8,6 \mathrm{~Hz}, \mathrm{H}-9)$ e $4,40(d d, \mathrm{~J}=6,9$ e $6,7 \mathrm{~Hz}, \mathrm{H}-9) ; 6,30$ $\left(s, \mathrm{H}-2^{\prime}\right) ; 6,30\left(s, \mathrm{H}-6^{\prime}\right) ; 4,58(d, \mathrm{~J}=4,2 \mathrm{~Hz}, \mathrm{H}-7 ') ; 2,67(d d$, $\mathrm{J}=12,0$ e $\left.2,7 \mathrm{~Hz}, \mathrm{H}-8^{\prime}\right) ; 5,93\left(\mathrm{sl}, \mathrm{O}_{2} \mathrm{C}\right) ; 3,61\left(2 \times \mathrm{CH}_{3} \mathrm{O}\right) ; 2,23$ $\left(\mathrm{OCCH}_{3}\right) ; 2,29\left(\mathrm{OCCH}_{3}\right)$. RMN de ${ }^{13} \mathrm{C}\left[75 \mathrm{MHz}, \mathrm{CDCl}_{3}, \delta\right.$ (ppm)]: $\delta$ 138,5 (C-1); 108,5 (C-2); 148,1 (C-3); 148,6 (C-4); 131,3 (C-5); 122,3 (C-6); 27,1 (C-7); 43,7 (C-8); 72,0 (C-9); 138,5 (C-1'); 107,6 (C-2'); 151,4 (C-3'); 127,7 (C-4'); 151,4 $\left(\mathrm{C}-5^{\prime}\right) ; 107,6\left(\mathrm{C}^{\prime} 6^{\prime}\right) ; 31,5\left(\mathrm{C}-7^{\prime}\right) ; 47,0$ (C-8'); 174,6 (C-9'); $56,2\left(2-\mathrm{OCH}_{3}\right) 102,3\left(\mathrm{O}_{2} \mathrm{CH}_{2}\right) ; 20,4$ e $168,7\left(\mathrm{OCOCH}_{3}\right) ; 20,5$ e $167,9\left(\mathrm{OCOCH}_{3}\right)$.

Ácido oleanólico (1a). Dados de RMN de ${ }^{13} \mathrm{C}$ comparados com os descritos na literatura ${ }^{7}$ (Tabela 1).

Ácido ursólico (2a). Dados de $\mathrm{RMN}$ de ${ }^{1} \mathrm{H}$ e de ${ }^{13} \mathrm{C}$ comparados com os encontrados na literatura ${ }^{7,8}$.

Ácido $2 \alpha, 3 \beta$-diidroxi-urs-12-en-28-ólico (5a). Dados de $\mathrm{RMN}$ de ${ }^{1} \mathrm{H}$ e de ${ }^{13} \mathrm{C}$ comparados com os obtidos na literatura ${ }^{7,8}$ (Tabela 1).

Ácido $2 \alpha, 3 \beta, 19 \alpha$-triidroxi-urs-12-en-28-ólico (6a). Dados de RMN de ${ }^{13} \mathrm{C}$ similares aos dos modelos utilizados ${ }^{7}$ são encontrados na Tabela 1.

$3 \beta$-Glicosil-sitosterol. Dados de RMN de ${ }^{13} \mathrm{C}$ comparados com os obtidos na literatura ${ }^{9}$

Teste de Letalidade de Artemia salina. Os ovos de camarão, Artemia salina e o sal marinho (Ocean Water - Alcon) foram adquiridos de uma loja de aquários local. O teste de letalidade empregado foi adaptado do método A descrito por Serrano e cols $^{5}$. Os ovos de Artemia salina foram eclodidos em um aquário retangular, utilizando-se água do mar artificial obtida de acordo com as especificações do fabricante. $\mathrm{O}$ sistema foi aerado com bomba específica. $\mathrm{O}$ aquário foi construído com uma divisão interna de acrílico contendo vários orifícios, produzindo dois compartimentos não equivalentes em tamanho. Os ovos foram adicionados no compartimento menor, previamente escurecido utilizando-se papel alumínio. O compartimento maior foi externamente iluminado com lâmpada $60 \mathrm{~W}$, de modo a atrair os camarões para este compartimento, após a eclosão. Os ovos foram incubados por 48 horas em temperatura ambiente. Foram preparadas soluções metanólicas de extratos $(5 \mathrm{mg} / \mathrm{mL})$, frações cromatográficas e substâncias puras $(200 \mu \mathrm{g} / \mathrm{mL})$ e adicionadas em frascos tipo "snap". O solvente foi eliminado com auxílio de pistola de aquecimento. Todos os experimentos, inclusive os de controle foram realizados em triplicata. Após 48 horas foram transferidos 10 camarões para cada frasco, utilizando pipeta Pasteur esterilizada e, o volume dos frascos completados até $5 \mathrm{~mL}$ com água do mar artificial. As amostras bem como os controles foram mantidos sob iluminação por mais 24 horas e o número de camarões sobreviventes foram determinados com auxílio de uma lupa. A larva era considerada morta caso não exibisse movimentos durante 10 segundos de observação. Para o estudo da citotoxicidade das substâncias puras ativas o teste foi realizado nas concentrações de 500, 50 e $10 \mathrm{ppm}$. Os dados obtidos foram processados e os valores de DL $_{50}$ apresentando $95 \%$ de confiança, foram calculados usando o método de probit ${ }^{10}$, o qual indica a toxicidade da amostra em termos da dose $\left(\mathrm{DL}_{50}\right)$, da concentração $\left(\mathrm{Cl}_{50}\right)$ e do tempo $\left(\mathrm{Tl}_{50}\right)$ necessários para matar $50 \%$ dos camarões em estudo. Além disto, a mortalidade foi corrigida empregando-se a fórmula de Abbott: $\mathrm{MC}=[\mathrm{MTe}(\%)-\operatorname{MTbr}(\%) / 100-$ MTbr $(\%)]$ 100, onde $\mathrm{MC}=$ mortalidade corrigida; $\mathrm{MTbr}=$ mortalidade do controle (branco) $\mathrm{MTe}=$ mortalidade do teste .

\section{RESULTADOS E DISCUSSÃO}

A identificação das lignanas $\mathbf{3}$ e $\mathbf{4}$ foi realizada em base aos dados de RMN de ${ }^{1} \mathrm{H}$ e de ${ }^{13} \mathrm{C}$ (PND, DEPT), ${ }^{1} \mathrm{H}-{ }^{1} \mathrm{H}$ COSY, ${ }^{1} \mathrm{H}^{1}{ }^{13} \mathrm{C}$ HETCOR, além de comparação com os dados espectrais encontrados na literatura ${ }^{2}$. As substâncias 3 e 4 mostraram conjunto de sinais característicos nos espectros de RMN consistentes com a natureza das lignanas do tipo aril-tetraidronaftaleno lactônicas (Parte Experimental). A comparação dos dados espectrais das substâncias 3 e $\mathbf{4}$ com os da literatura ${ }^{2}$ sugeriram que estes eram semelhantes aos das lignanas $\beta$-peltatina e $\alpha$ peltatina, respectivamente. Os assinalamentos corretos dos deslocamentos químicos e a confirmação da estrutura da substância 4 foi efetuada por meio das correlações encontradas nos espectros de ${ }^{1} \mathrm{H}-{ }^{1} \mathrm{H}$ COSY e ${ }^{1} \mathrm{H}^{-13} \mathrm{C}$ HETCOR (direto e à longa distância), bem como pela análise dos espectros de RMN (monodimensional e bidimensional) obtidos para o derivado acetilado (4a). Desta forma, as correlações observadas entre $\mathrm{H}-2$ com C-3 e C-4, bem como entre H-2' e H-6' com C-1', C-3' e C-4', encontradas nos espectros ${ }^{1} \mathrm{H}_{-}{ }^{13} \mathrm{C}$ HETCOR à longa distância $\left({ }^{3} \mathrm{~J}=7 \mathrm{~Hz}\right)$ de 4 e do seu derivado acetilado (4a) corroboraram uma estrutura semelhante à $\alpha$-peltatina para a substância 4 . Os espectros de massas destas duas substâncias mostraram o sinal do pico do íon molecular em $\mathrm{m} / \mathrm{z} 414 \mathrm{e} \mathrm{m} / \mathrm{z}, 400$ coerentes com as fórmulas moleculares $\mathrm{C}_{22} \mathrm{H}_{22} \mathrm{O}_{8}$ e $\mathrm{C}_{21} \mathrm{H}_{20} \mathrm{O}_{8}$ observadas para a $\beta$-peltatina e $\alpha$-peltatina, respectivamente.

As lignanas $\alpha$-peltatina e $\beta$-peltatina, também encontradas em Podophyllum peltatum ${ }^{2}$ pertencem ao grupo da podofilotoxina e possuem atividade citotóxica conhecida. A presença destas lignanas também foi relatada no único trabalho desenvolvido anteriormente com este gênero realizado com Eriope macrostachya ${ }^{11}$. Portanto, a atividade citotóxica in vitro observada no extrato clorofórmico da espécie pode ser devido à presença dessas lignanas. Como comprovação desta suposição todas as substâncias isoladas foram submetidas ao teste de letalidade de Artemia salina, pois é conhecida a existência de uma correlação entre a toxicidade das substâncias sobre as larvas de camarão recém eclodidas e a atividade citotóxica ${ }^{12}$. A Tabela 3 apresenta os resultados de toxicidade observados para os compostos isolados de E. blanchetii. Pelos dados observa-se que a substância responsável pela atividade pode ser a $\alpha$-peltatina e em bem menor extensão a $\beta$-peltatina. É digno de nota que o derivado acetilado (4a) apresenta atividade muito inferior a observada para 4, demonstrando assim a importância do grupo hidroxílico livre no C-4' para a atividade. De acordo com a literatura ${ }^{12}$ a substância que apresentar $\mathrm{LD}_{50} \leq 100 \mu \mathrm{g} / \mathrm{mL}$ é comparável com a camptotecina e sulfato de vincristina, sendo considerada muito ativa e, aquela que apresentar $\mathrm{DL}_{50} \geq 100 \mu \mathrm{g} / \mathrm{mL}$, no intervalo entre 100 e $900 \mu \mathrm{g} / \mathrm{mL}$, comparável ao ácido hipúrico é considerada medianamente ativa. Enquanto que é considerada inativa a substância que apresentar $\mathrm{LD}_{50}>1000 \mu \mathrm{g} / \mathrm{mL}$.

A elucidação estrutural dos ésteres metílicos dos ácidos oleanólico (1a), ursólico (2a), $2 \alpha, 3 \beta$-diidroxi-urs-12-en-28-ólico (5a) e bem como do $3 \beta$-glicosil sitosterol, foi realizada por meio de comparação direta com os dados descritos na literatura ${ }^{7,8}$. Por outro lado, a determinação estrutural do éster metílico derivado do ácido $2 \alpha, 3 \beta, 19 \alpha$-triidroxi-urs-12-en-28-ólico, ou ácido tormentico (6a), foi realizada usando diferentes modelos encontrados na literatura, haja vista que embora sendo conhecido não foram encontrados dados de $\mathrm{RMN}$ de ${ }^{13} \mathrm{C}$ descritos. 
Tabela 3. Dados de letalidade de Artemia salina para as substâncias isoladas de E. blanchetii $\left(\mathrm{DL}_{50} \mathrm{em} \mu \mathrm{g} / \mathrm{mL}\right)$

\begin{tabular}{cc}
\hline Substância & DL $_{50}$ \\
\hline $\mathbf{1}$ & $>1000$ \\
$\mathbf{2}$ & $>1000$ \\
$\mathbf{3}$ & 900 \\
$\mathbf{4}$ & 10 \\
$\mathbf{4 a}$ & $>1000$ \\
$\mathbf{5 a}$ & $>1000$ \\
$\mathbf{6 a}$ & $>1000$ \\
\hline
\end{tabular}

\section{CONCLUSÕES}

Neste trabalho foram isolados quatro triterpenos pentacíclicos, sitosterol glicosilado, bem como $\beta$-peltatina e $\alpha$-peltatina, lignanas responsáveis pela atividade citotóxica observada nos testes in vitro. Cerca de $30 \%$ do extrato $\mathrm{CHCl}_{3}$ é formado pelo ácido oleanólico, portanto a pequena expressão desta atividade citotóxica no extrato bruto, provavelmente, é decorrente da alta concentração dos ácidos triterpênicos, especialmente do ácido oleanólico.

\section{AGRADECIMENTOS}

Os autores são gratos à FINEP e ao Conselho Nacional de Desenvolvimento Científico e Tecnológico (CNPq) pelo auxílio financeiro e pelas bolsas, bem como ao PIBIC/CNPq/UFBA pelas bolsas de Iniciação Científica. Também agradecem ao Dr. Geoffrey A. Cordell da University of Illinois at Chicago pelos testes in vitro do extrato de E. blanchetii.

\section{REFERÊNCIAS}

1. Hardman, J. G.; Limbird, L. E.; Molinoff, P. B.; Ruddon, R. W.; Gilman, A. G. Eds. In Goodman \& Gilman's The Pharmacological Basis of Therapeutics; 9th Edition; McGraw-Hill: New York, 1996; pp. 1225-1287.

2. Ayres, D. C.; Loike, J. D. Lignans: Chemical, Biological and Properties; Cambridge University Press; New York, 1990.

3. Harley, R. M.; Simmons, N. A. Flora of Mucugê, Chapada Diamantina, Bahia, Brazil. Royal Botanic Garden, Kew, 1986.

4. Harley, R. M.; Hooker's Icones Plantarum 1976, XXXVIII, 1.

5. Serrano, C.; Ortega, T.; Villar, A. M.; Phytother. Res. 1996, 10, S118.

6. Likhitwitayawuid, K.; Angerhofer, C. K.; Cordell, G. A.; Pezzuto, J. M.; Ruangrungsi, N.; J. Nat. Prod. 1993, 56, 30.

7. Mahato, S. B.; Kundu, A. P.; Phytochemistry 1994, $37,1517$.

8. Romeo, G.; Gianetto, P.; Aversa, M. C.; Org. Magn. Reson. 1977, 9, 29.

9. Matida, A. K.; Rossi, M.; Almeida Blumenthal, E. E.; Schuquel, I. T. A.; Malheiros, A.; Vidotti, G. J.; An. Ass. Brasil. Quím. 1996, 45, 147.

10. Carazo, E.; Martínez, J. L.; Bustamante, M. In Metodologias para el Estudio y Manejo de Moscas Blancas y Geminivirus; Hilge, L., Ed.; Catie Unidad de Fitoprotección, Turrialba, Costa Rica, 1996; pp. 89-91.

11. Raffauf, R. F.; Kelley, C. J.; Ahmad, Y.; Le Quesne, P. W.; J. Nat. Prod. 1987, 50, 772.

12. Anderson, J. E.; Goetz, C. M.; McLaughlin, J. L.; Suffness, M.; Phytochem. Anal. 1991, 2, 107. 